\title{
Additional evidence from a paradigm for inferring discriminative processes in children and adults
}

\author{
ELIZABETH S. GHATALA \\ Weber State College, Ogden, Utah 84408 \\ JOEL R. LEVIN \\ University of Wisconsin, Madison, Wisconsin 53706 \\ and \\ KIM P. WILSON \\ Weber State College, Ogden, Utah 84408
}

\begin{abstract}
A previously developed paradigm which incorporates a correlational methodology into an experimental context was used in the present study to specify more definitely the attribute(s) underlying verbal discrimination learning under normal conditions. In the case of fifth- and sixth-grade children, it was found that frequency theory is sufficient to account for performance on the task. However, older subjects were less homogeneously reliant on the frequency attribute. While many older subjects appeared to utilize frequency cues, others adopted strategies that resulted in the utilization of attributes other than frequency.
\end{abstract}

In two recent studies (Ghatala, Levin, \& Subkoviak, 1975; Levin \& Ghatala, 1976), we have utilized Underwood's (1975) individual differences approach to uncover the functional components underlying children's verbal discrimination learning. This has been achieved both under conditions where subjects are left to their own devices in performing the task (i.e., the usual or "control" version of the task) and under conditions where subjects are instructed to employ a facilitative rehearsal strategy. The results of our previous work indicate that the mechanisms underlying performance on the task are quite different in the two situations.

By way of summarizing this research, our paradigm requires that subjects in the control and strategy conditions perform three tasks. The first is a relative frequency judgment task designed to measure subjects' ability to discriminate situational frequency differences between verbal items. The second is the target task, verbal discrimination learning, and the third is a strategy identification task designed to determine how well

This research was funded by the Wisconsin Research and Development Center for Cognitive Learning, supported in part as a research and development center by funds from the National Institute of Education (Center No. NE-C-00-3-0065). The opinions herein do not necessarily reflect the position or policy of the National Institute of Education. We are grateful to the participating staffs and students of Grandview and Horace Mann Elementary Schools and Ben Lomond High School in Ogden, Utah for their gracious cooperation in the research, and to Marion Bushell for typing the paper. The first two authors contributed equally to the study. Reprint requests should be addressed to Elizabeth S. Ghatala, who is now at the Foundations of Education Department, University of Houston, Houston, Texas 77004. Kim Wilson is now at Northwestern University.

subjects can discriminate items for which they had been requested to employ a particular rehearsal strategy (e.g., pronunciation) from items for which they had not. In addition, there are two between-subjects conditions reflecting the two discrimination-learning variations mentioned at the outset. That is, in the control condition, subjects receive no special rehearsal instructions for verbal discrimination learning, whereas in the strategy condition they are requested to employ a rehearsal strategy for verbal discrimination learning (the same one used in the strategy identification task, e.g., pronunciation). The pattern of correlations among the three tasks in the control condition is then contrasted with the pattern in the strategy condition.

This paradigm has now been applied to four different kinds of rehearsal strategy: pronunciation and imagery (Ghatala et al., 1975) and rhyme and function (Levin \& Ghatala, 1976). In each case the control condition produced the same results, namely, a significant correlation of around .40 between frequency judgment ability and verbal discrimination learning but no correlation between strategy identification ability and verbal discrimination learning. However, the correlational patterns produced in the strategy conditions were quite different from the control pattern. For strategies such as imagery, pronunciation, and rhyming (which have been shown not to positively affect simple frequency processes), there was à significant correlation (ranging from .60 to .70) between strategy identification ability and verbal discrimination learning but no correlation between frequency judgment ability and verbal discrimination learning. For the function strategy (which enhances performance on frequency judgment tasks), both strategy identification ability and frequency 
judgment ability were significantly correlated (each about .40) with verbal discrimination learning. The function strategy requires subjects to tell what the object referent of the correct response does (e.g., "sweep" would be a function response for the item broom).

We concluded from these patterns that frequency is the dominant attribute underlying performance in verbal discrimination learning under control conditions, but that frequency is supplanted by an "activity" attribute (as measured by the strategy identification task) in the case of the imagery, pronunciation, and rhyming strategies, and supplemented by this attribute in the case of the function strategy. In other words, frequency theory (Ekstrand, Wallace, \& Underwood, 1966) can account for children's verbal discrimination learning under normal (i.e., control) conditions, but the theory is not sufficient to account for the facilitative effects of various rehearsal strategies on verbal discrimination learning. While the evidence for the second half of this conclusion appears unequivocal, the evidence for the first half, when considered in the context of certain issues about the control pattern raised by Levin and Ghatala (1976), may seem less convincing. The primary purpose of the present research was to specify with more certainty the attribute(s) underlying verbal discrimination learning in the control condition.

If, as we have argued, frequency is the dominant attribute utilized by control subjects, one must wonder why the correlation between frequency judgment ability and verbal discrimination learning is not higher. The .40 correlation between the frequency judgment task and the verbal discrimination task consistently found in the control condition certainly does not rival the .60 to .70 correlations found between the strategy identification and verbal discrimination tasks in the imagery, pronunciation, and rhyme strategy conditions, where we also infer that a single, though different, attribute underlies performance.

Two explanations-apart from reliability considerations mentioned by Levin and Ghatala (1976)-can plausibly account for the comparatively lower correlation between the frequency judgment task and verbal discrimination learning in the control condition. First, even though frequency is materially involved in both tasks, the kind of frequency encoding required of subjects differs for each. In both the Ghatala et al. (1975) and Levin and Ghatala (1976) studies, the instructions given for the relative frequency judgment task did not specify the nature of the later test, whereas, in the discrimination learning task, subjects were explicitly told what would be expected of them on the test trial. Thus, if subjects were encoding item frequencies, they were likely doing so in an incidental mannner for the former situation and in a much more intentional manner for the latter situation. While it is true that a number of previous researchers have found that explicit instructions to encode frequency do not seriously affect the accuracy of adults' frequency judgment performance in comparison to performance under nonexplicit instructions (e.g., Flexser \& Bower, 1975; Rose \& Rowe, 1976), there may well be individual differences with respect to subjects' ability to encode frequencies intentionally, as opposed to incidentally. These differences would be reflected in a correlational analysis, though not necessarily in a comparison of group means. An implication of this reasoning is that the correlation between frequency judgment ability and verbal discrimination learning would increase were the relative frequency judgment task administered under intentional instructions.

It is also plausible that attributes other than frequency are involved in the task under control conditions but that our paradigm as presently constituted is insensitive to them. That is, we have noted that strategy identifications involving imagery, pronunciation, rhyming, and generating functions are not related to control verbal discrimination performance. But it is important to note that this conclusion is based on the forced usage of a fixed strategy in the strategy identification task. If different subjects tend to employ different (likely idiosyncratic) covert strategies in the discrimination task, there is no reason to anticipate a correlation between strategy identification ability based on any specified strategy and discrimination learning. On the other hand, if subjects were encouraged to adopt whatever strategy they wanted to in a strategy identification format, performance under this condition might be expected to predict control discrimination learning. In Experiment 1, we gathered evidence related to both of these speculations.

\section{EXPERIMENT 1}

\section{Method}

Experimental design and predictions. An overview of the design and sequence of events is presented in Table 1 . Subjects within both conditions of the experiment (unstructured-control and pronunciation-strategy) were administered three tasks utilizing different verbal items (all printed nouns). Task 1 (relative frequency judgments) measured subjects' ability to make accurate frequency discriminations. The same version of this task (which was given here under intentional instructions as opposed to the nonspecific instructions utilized by us previously) was received by subjects in both conditions. Task 2 was verbal discrimination learning, the target phenomenon of the study. For this task, subjects in the unstructured-control condition were not given any rehearsal strategy instructions, while subjects in the pronunciation-strategy condition were instructed to rehearse correct items by pronouncing them aloud. Finally, in Task 3 (strategy identification) subjects were shown a list of words and asked to rehearse half of them. Subjects in the unstructured-control group were encouraged to employ any

Table 1 Design of the Experiment

\begin{tabular}{cccc}
\hline Condition & Week 1 & Week 2 & Week 3 \\
\hline Unstructured-Control & RFJ & VDL* & SI** \\
Pronunciation-Strategy & RFJ & VDL $\dagger$ & SI \\
\hline
\end{tabular}

Note-RFJ = relative frequency judgment task, VDL = verbal discrimination learning, $S I=$ strategy identification task *Contro! **Unstructured +Pronunciation 
covert rehearsal strategy they wished, while subjects in the pronunciation-strategy group were required to employ a pronunciation rehearsal strategy. Upon re-presentation of the words in a different order, subjects were required to discriminate the items they had been asked to rehearse from those they had not.

All tasks were administered in the same temporal order to all subjects since the intent of the study was to examine intertask correlations under different instructional conditions, a situation in which it is not useful to counterbalance tasks (see Levin \& Ghatala, 1976). Moreover, the particular order of task administration allowed the two predictor tasks (relative frequency judgments and strategy identification) to be temporally equidistant from the criterion task (verbal discrimination learning) and also minimized potential intertask reactivity as related to the particular instructions. A 1-week interval between tasks was also employed to deal with the latter concern.

The predictions concerning intertask relationships in the pronunciation-strategy group are straightforward. That is, based on previous results with this particular strategy (Ghatala et al., 1975 ) and others of the same class (Ghatala et al., 1975; Levin \& Ghatala, 1976), one would expect a substantial correlation (of .60 to .70 ) between the strategy identification task and verbal discrimination learning but little correlation between the frequency judgment task and verbal discrimination learning. This correlational pattern, which we have labeled the supplant pattern, indicates that when the strategy is employed during discrimination learning, strategy discriminations rather than frequency discriminations are involved in performing the task. The pronunciation-strategy condition was included to provide a known baseline against which to compare the results obtained in the unstructured-control condition where, given certain assumptions, several patterns are plaúsible.

First of all, if frequency discrimination is actually the predominant process underlying performance in verbal discrimination learning in the absence of rehearsal strategy instructions, then one would expect the same pattern as that obtained for control conditions in previous experiments, namely, a significant correlation between frequency judgment ability and discrimination learning but no correlation between strategy identification ability and discrimination learning. Moreover, if the frequency encoding that occurs in the control verbal discrimination learning condition is reflected more accurately by intentional than by incidental frequency judgments, then the correlation between performance on the present intentional frequency judgment task and discrimination learning should be more substantial than the .40 value found in our previous work. This result would imply that frequency theory is sufficient to account for children's verbal discrimination learning under normal conditions.

On the other hand, if some subjects tend to employ covert rehearsal strategies that result in the generation and utilization of cues other than frequency, and if the unstructured strategy identification task is a valid measure of subjects' ability to do this, then one would expect verbal discrimination learning to be correlated with both the unstructured strategy identification and relative frequency judgment tasks (the supplement pattern). This result would imply that, although frequency plays a role in children's discrimination performance (as evidenced by our previous findings), it is not the sole attribute involved.

Subjects. The 66 subjects were the same age (fifth-and six thgrade children) and from the same community (Ogden, Utah) as those employed in our previous research. Children were randomly assigned to the two conditions (unstructured-control and pronunciation-strategy) in the order of their appearance at the testing room located within the school building. Thus, 33 children (nearly equally divided between grades) participated in each condition.

Materials, tasks, and procedures. From an initial pool of 208 concrete nouns, 80 words were randomly selected for use in the relative frequency judgment task, 48 for the verbal discrimination task, and 80 for the strategy identification task. The three sets of materials were comparable in Thorndike-Lorge frequency, with almost half of the items in the AA or A range. All words were in the reading vocabularies of the children.

Subjects were tested individually on the relative frequency judgment task first; then 1 week later, on the verbal discrimination task; and after another 1-week interval, on the strategy identification task. The children were told that they would participate on three occasions and that the tasks would be unrelated.

Relative frequency judgment task. In this task, items presented a different number of times during study were paired on the test trial and subjects were required to choose the more frequent member of each pair. On the test trial there were 10 one vs two pairs (i.e., subjects were required to discriminate between items presented once and items presented twice during study). There were also 10 one vs three pairs, 10 two vs three pairs, and 10 two vs four pairs on the test. Achieving the necessary induced frequencies required 180 study presentations: 20 words were presented once; 30 , twice; 20 , three times; and 10 , four times. All words were randomly assigned to the four presentation frequencies.

The ordering of words across the 180 study positions was random, subject to the restriction that those with multiple occurrences appeared equally often in each equal-sized section of the list. The same word never occurred in adjacent positions. The words were typed on $5 \times 8$ in. plain white cards which were fastened into a ringed binder. The four types of test pairs were constructed by randomly pairing items from the four frequency categories. The order of the pairs on the test was random. The words in the test pairs were typed side by side, on $5 \times 8$ in. cards which were fastened into a notebook. The more frequent words appeared equally often in the left- and right-hand positions across pairs.

The procedure for the relative frequency judgment task was identical in the two experimental conditions. Specifically, and in contrast to our previous experiments in which precise information about the nature of the task was not given, the instructions in the present experiment described the frequency judgment task for which subjects were to prepare themselves. The words were presented for study at a 3-sec rate. The same rate was used on the test trial, with subjects pointing to the more frequent word in each pair, guessing if uncertain.

Verbal discrimination task. The 48 words were randomly paired to form a 24-pair list, with the correct member of each pair being determined by the flip of a coin. The word pairs were typed on $5 \times 8$ in. cards which were placed in a Rolodex file The task consisted of one study trial (two items presented, followed by feedback) and one anticipation-test trial. On the feedback portion of each trial the correct item in each pair was designated by a plus sign underneath it. A different random order of the list was used on the two trials. The spatial position of correct and incorrect items within pairs was arranged such that: (1) on each presentation of the list, correct and incorrect items occurred equally often in the left and right positions, and (2) for half of the pairs the position of the correct item changed from the study to the test trial.

The procedure for the verbal discrimination task varied as a function of experimental condition. Subjects in the unstructuredcontrol condition received the usual instructions for the present task. In addition to these instructions, subjects in the pronunciation-strategy group were instructed to pronounce the correct item three times during the feedback portion of the study trial. On the test trial, all subjects were required to point to the correct member of each pair (guessing if uncertain) during the anticipation phase. A 4-sec rate was utilized for study and test trials in both conditions.

Strategy identification task. Of the 80 words presented individually for study, 40 were randomly selected as strategy items. Subjects were instructed to apply the appropriate rehearsal strategy only to items that were underlined on the study trial. Subjects in the pronunciation-strategy group were told to pronounce aloud each underlined word three times. Subjects in 
the unstructured-control group were told to do some special thing silently that would help them remember the underlined words. All subjects were informed as to the nature of the later test. On the test, the same words were presented in a different random order, with none underlined. Subjects said "yes" if they thought they had previsouly applied the rehearsal strategy to a particular word and "no" if they thought they had not previously applied it. Subjects responded for every item, guessing if uncertain. Both the study and test trials proceeded at a 3-sec rate.

For all tasks, the experimenter presented the materials by turning cards; thus, the above rates of presentation are approximate. However, the experimenter was well practiced and a stopwatch was used to check rates periodically throughout the course of the experiment.

\section{Results and Discussion}

Analysis of mean performance. As in our previous studies, prior to inspecting the intertask correlations within each condition, mean performance was examined to verify the adequacy of random assignment procedures and the anticipated facilitatory effect of the pronunciation rehearsal strategy on verbal discrimination learning. With regard to the first concern, the two groups did not differ on the relative frequency judgment task, indicating that randomization procedures resulted in equal ability groups on this measure. The means were $30.7(\mathrm{SD}=4.2)$ and $31.6(\mathrm{SD}=3.9)$ correct out of 40 for the unstructured-control and pronunciationstrategy groups, respectively $[|t|(64)<1]$.

Conceming strategy effects in the verbal discrimination task, pronunciation (mean of 19.8 out of 24 , $\mathrm{SD}=2.5$ ) clearly facilitated performance relative to the unstructured-control group [mean of $16.1, \mathrm{SD}=3.0$, $t(64)=5.41, p<.01]$, thereby confirming the results of previous research (e.g., Ghatala et al., 1975; Levin, Ghatala, DeRose, Wilder, \& Norton, 1975; Levin, Ghatala, Wilder, \& Inzer, 1973).

Examination of the correlational patterns. The intercorrelations among the three tasks are presented in Table 2 for the two conditions. Starting first with the correlational pattern of the pronunciation-strategy condition, one will note its complete agreement with previous results for this and other strategies of the same type. That is, strategy identification ability but not frequency judgment ability is significantly related to verbal discrimination learning, with the magnitude of the strategy identification/discrimination learning correlation virtually identical to that reported by Ghatala et al. (1975) for the pronunciation strategy. Moreover, frequency judgment ability and strategy identification

Table 2

Intertask Correlations in the Two Conditions of Experiment 1

\begin{tabular}{|c|c|c|c|c|}
\hline & \multicolumn{2}{|c|}{ Unstructured-Control } & \multicolumn{2}{|c|}{ Pronunciation-Strategy } \\
\hline & RFJ & & RF J & \\
\hline SI & .05 & & .09 & \\
\hline VDL & $.62 *$ & .11 & .13 & $.63 *$ \\
\hline
\end{tabular}

Note $-R F J=$ relative frequency judgment task, $V D L=$ verbal discrimination learning, SI = strategy identification task ${ }^{*} p<.01$ ability are statistically uncorrelated, a result also obtained earlier (Ghatala et al., 1975; Levin \& Ghatala, 1976).

In the unstructured-control condition, the correlational pattern is quite different from the now wellsubstantiated pronunciation-strategy pattern. Here, frequency judgment ability is significantly related to verbal discrimination learning but strategy identification ability is not. This is the typical control pattem found by Ghatala et al. (1975) and Levin and Ghatala (1976) and would appear to indicate that, indeed, frequency is the predominant attribute in children's verbal discrimination learning under normal circumstances. The preceding conclusion is supported by the magnitude of the correlation between frequency judgment ability and verbal discrimination learning in the unstructuredcontrol condition $(r=.62)$, which is substantially greater than the expected .40 value as determined from our former control conditions $(p<.05$, one-tailed). For reasons outlined in the introduction, we tend to attribute the increase in the magnitude of this correlation to the use of intentional rather than nonspecific instructions in the relative frequency judgment task. Although this conclusion is tentative, since there has been no direct test of it, it appears quite reasonable in the absence of plausible alternative explanations.

The level of performance on the strategy identification task obtained by subjects in the unstructuredcontrol condition (mean of 49.2 out of $80, \mathrm{SD}=6.7$ ) was lower than that obtained by subjects in the pronunciation-strategy condition [mean of 61.8, $\mathrm{SD}=5.9, \mathrm{t}(64)=8.08, \mathrm{p}<.01]$. While performance in the former condition was statistically above chance-indicating that subjects could make reliable activity discriminations in this task-the basis for discrimination is unclear. Moreover, subjects were not very good at describing the "special thing" they did to remember which words were underlined. Of the 11 subjects who could verbalize what they had done on the task, 9 reported saying the underlined words silently, 1 reported using imagery, and 1 reported adopting a serial order strategy.

In conclusion, the results of Experiment 1 indicate that frequency theory is sufficient to account for verbal discrimination learning under normal circumstances, at least for the elementary school aged subjects that we have tested in this and previous experiments. Whether or not this conclusion can be generalized to older subjects who may be more likely to employ rehearsal strategies spontaneously in the verbal discrimination task (e.g., Rowe \& Cake, 1974) is the question addressed in the next experiment.

\section{EXPERIMENT 2}

\section{Method}

The design, tasks, and procedures were exactly the same as in the first experiment. The subjects were 60 students attending 
high school in Ogden. The subjects were selected from among college-bound juniors and seniors and were between 17 and 18 years of age. Subjects were randomly assigned in equal numbers to the unstructured-control and pronunciation-strategy conditions and were individually tested in a private room in the school building.

\section{Results and Discussion}

Analysis of mean performance. As before, "validity" checks were conducted to verify the adequacy of random assignment and the effectiveness of the pronunciation strategy in discrimination learning. The virtually identical performance on the relative frequency judgment task by the unstructured-control group (mean correct of $31.4, \mathrm{SD}=3.6$ ) and the pronunciationstrategy group $[$ mean $=31.6, \quad \mathrm{SD}=3.2,|\mathrm{t}|(58)<1]$ affirms that randomization procedures resulted in equal ability groups. Concerning the strategy effect in discrimination learning, pronunciation (mean of 20.9, $\mathrm{SD}=2.5$ ) clearly facilitated performance relative to the unstructured-control group [mean of 17.4, $\mathrm{SD}=3.2, \mathrm{t}(58)=4.64, \mathrm{p}<.01]$. This result is in accord with numerous studies showing the facilitative effect of pronunciation on adult subjects' verbal discrimination performance (e.g., Rowe \& Paivio, 1971; Underwood \& Freund, 1968; Wilder, 1971).

Examination of the correlational patterns. The correlations among tasks within each condition are shown in Table 3. Considering the pronunciation-strategy condition first, one can see that strategy identification ability rather than frequency discrimination ability is the prime predictor of verbal discrimination performance. Thus, with adult subjects as well as with children, when a rehearsal strategy such as pronunciation is utilized in the verbal discrimination paradigm, the task does not seem to depend on the formation of subjective frequency differentials between correct and incorrect pair members. Rather, performance in the task appears to involve discriminations between items on the basis of strategy-generated activity cues as measured by the strategy identification task (see Ghatala et al., 1975, for further discussion on the nature of the so-called activity attribute).

The correlational pattern exhibited by the unstructured-control group is different from any pattern we have previously obtained. At first glance it resembles the supplement pattern found by Levin and Ghatala (1976) for the function strategy, which, the reader will recall, requires subjects to tell what the object referent

Table 3

Intertask Correlations in the Two Conditions of Experiment 2

\begin{tabular}{lcccc} 
& \multicolumn{2}{c}{ Unstructured-Control } & \multicolumn{2}{c}{ Pronunciation-Strategy } \\
RFJ & SI & RFJ & SI \\
\hline SI & $.38^{*}$ & & .14 & \\
VDL & $.45^{*}$ & $.72 * *$ & .19 & $.71^{* *}$ \\
\hline
\end{tabular}

Note $-R F J=$ relative frequency judgment task, $V D L=$ verbal discrimination learning, $S I=$ strategy identification task ${ }^{*} p<.05$

$* * p<.01$ of each correct item does. The resemblance resides in the finding that both the frequency judgment task and the strategy identification task are significantly correlated with verbal discrimination learning. However, there are two important aspects of these data that render it different from the function strategy's supplement pattern: (1) In the present case, discrimination learning appears to be better predicted from the strategy identification task $(r=.72)$ than from the frequency judgment task $(r=.45)$, whereas in the Levin and Ghatala situation, discrimination learning was predicted equally by the two $(r=.40) ;(2)$ in the present case, the two predictor variables are significantly correlated $(\mathrm{r}=.38)$, whereas previously they were not $(\mathrm{r}=.03)$.

Additional analyses. Point 1 above was examined in two related ways. First, correlations were computed between each predictor task and the criterion task, with the effect of the other predictor task held constant. When this was done, the partial $r$ between strategy identification ability and discrimination learning (.66) remained significant $(p<.01)$, whereas that between frequency judgment ability and discrimination learning (.26) no longer was $(\mathrm{p}>.10)$. Second, the two predictor variables were entered into multiple regression equations (see Levin \& Ghatala, 1976) in both possible orders. When frequency judgment ability was entered first, the additional discrimination learning variance accounted for by strategy identification ability (35\%) was statistically significant. On the other hand, when strategy identification ability was entered first, statistically no additional discrimination learning variance $(3 \%)$ was explained. Both of these analyses suggest that in the adult unstructured-control condition, the strategy identification task is clearly more predictive of discrimination learning than is the frequency judgment task, a situation quite the reverse of the children's unstructured-control pattern revealed in Table 1 . This is not to deny the possibility that frequency processes are involved in the adult discrimination learning situation as well. Indeed, in the following analyses we entertain the notion that there exist individual differences in attribute selection that must be considered.

Originating from Point 2 above, one can reasonably posit that the strategy identification task (in its unstructured format) is not entirely independent of frequency discrimination processes. Indeed, in our earlier work (Ghatala et al., 1975) we rejected the notion that strategy identification performance could be accounted for in frequency-theory terms, since such an account (in the face of a consistent lack of correlation between the frequency judgment and strategy identification tasks) put a great deal of strain on the present version of the theory. However, it makes some sense to pursue this argument here (certainly as opposed to a complicated converse argument that attributes frequency judgment performance to strategy identification ability). In this regard, then, the tactics that many of the subjects reported using in the strategy identification task seemed 
designed specifically to build up a subjective frequency differential between underlined and nonunderlined words. In particular, when asked to describe what they did in order to remember which words had been underlined on the strategy identification task, 17 (out of 30 ) subjects mentioned the following activities: (1) paying attention to the underlined words, (2) repeating the underlined words silently, and (3) ignoring the nonunderlined words. Some of these 17 subjects reported doing only one of these activities but most reported that they paid attention to (or silently repeated) the underlined words and ignored the nonunderlined words.

On the other hand, the activities reported by a smaller subset of seven subjects resembled a number of the experimenter-instructed strategies that we have previously examined. That is, two subjects reported that they thought of images for the underlined words (Ghatala et al., 1975) and five said that they constructed either verbal associates or sentences for them (Levin \& Ghatala, 1976). If these spontaneously employed strategies operate like the experimenter-instructed ones, then the discrimination of underlined from nonunderlined words for these subjects should be based on distinctive activity cues rather than on subjective frequency differentials.

Assuming that our interpretation of the discriminative processes indicated by various kinds of selfreported activities on the strategy identification task is valid, then we can classify subjects into two subgroups: frequency encoders and activity encoders. In theory, subjects who simply repeat the underlined items to themselves could be classified as activity encoders; however, as was noted above, in practice, repetition subjects typically reported also engaging in frequency encoding activities (i.e., selective attending and ignoring). Six subjects who failed to respond when questioned about their activities on the strategy identification task were not included in the following analysis.

If it can further be assumed that groupings are not associated with relevant self-selected preferences or abilities (the latter is discussed below), then it might be expected that frequency judgment ability would predominate in the case of frequency encoders, and strategy identification ability would predominate in the case of activity encoders. This is essentially what we found. As may be seen in Table 4, for the 17 so-called frequency encoders, all three tasks are significantly correlated. The most straightforward explanation of this pattern focuses on frequency as the primary attribute underlying performance in all three tasks. In contrast, for activity encoders only the strategy identification/discrimination learning correlation is nontrivial (and impressively so), suggesting that their discrimination learning performance can be characterized almost exclusively by activity discriminations.

It is interesting to note that the two subgroups did not differ in mean performance on any of the three tasks [all $|t| s(22)<1]$, which indicates that: (1) the division of subjects on the basis of reported strategy
Table 4

Intertask Correlations for Frequency and Activity Encoders in the Unstructured-Control Condition of Experiment 2

\begin{tabular}{lllll}
\hline & \multicolumn{2}{c}{$\begin{array}{c}\text { Frequency } \\
\text { Encoders } \\
(\mathrm{N}=17)\end{array}$} & \multicolumn{2}{c}{$\begin{array}{c}\text { Activity } \\
\text { Encoders } \\
(\mathrm{N}=7)\end{array}$} \\
& RFJ & SI & RFJ & SI \\
\hline SI & $.61^{*}$ & & .05 & \\
VDL & $.65^{*}$ & $.78^{*}$ & .15 & $.89^{*}$ \\
\hline
\end{tabular}

Note-RFJ $=$ relative frequency judgment task, $V D L=$ verbal discrimination learning, $S I=$ strategy identification task $* p<.01$

utilization is not confounded by differences in frequency discrimination ability, and (2) the spontaneous utilization of different attributes by subjects on the verbal discrimination task (as inferred from the different correlational patterns associated with the two subgroups) is not accompanied by mean performance differences on these tasks.

We are inclined to argue on the basis of the above analyses that for adult subjects there is no general answer to the question, "What attributes are utilized in verbal discrimination learning under normal circumstances?" Rather, some subjects appear to utilize frequency cues, while others adopt strategies that result in the utilization of attributes other than frequency.

\section{EXPERIMENT $2 a$}

This conclusion must be viewed with caution for a number of reasons. First, the numbers of subjects involved in the correlations within each subgroup were relatively small. Second, subjects were classified into the subgroups on the basis of a post hoc interpretation of their reported activities on the strategy identification task. With regard to this latter concern, subjects were classified as activity encoders according to a relatively simple criterion, namely, the reported usage of a rehearsal strategy that elaborated upon or added to the stimulus item (e.g., imagery or elaborative verbal responses). Happily, comparing the correlational pattern of these subjects with those in the experimenterinstructed pronunciation-strategy condition in Table 3 , one will note an obvious correspondence. However, the clessification of subjects as frequency encoders was more complex and involved intuitive judgments that the various activities (i.e., paying attention to items, silently repeating items, ignoring items), reported singly or in combination by subjects, indicated the utilization of subjective frequency as a basis for discrimination in the strategy identification task. In fact, this may have been an erroneous interpretation despite the meaningful correlational pattern obtained for these subjects. Or, even if our interpretation of subjects' activities was essentially correct, the problem of potential selfselection (alluded to earlier) prevents our attributing the correlations in question to the operation of frequency encoding processes per se. Fortunately, we 
were able to test the validity of our interpretation of frequency encoding activities and, at the same time, overcome the problem of subject selection.

\section{Method}

To do this, we obtained an additional sample of 30 subjects from the same high school population. These subjects were administered the relative frequency judgment task just as in the main experiment. In their second session, subjects were administered the strategy identification task with instructions that incorporated the activities reported by subjects who we classified as frequency encoders in the main experiment. That is, subjects were instructed to pay attention to the underlined words and to ignore the nonunderlined words. It was further suggested to the subjects that they might want to repeat the underlined words silently while paying attention to them.

\section{Results and Discussion}

The correlation between the strategy identification task and the relative frequency judgment task obtained for this new group was $.46(\mathrm{p}<.01)$, a value slightly smaller than, though not statistically different from, the .61 correlation associated with the 17 frequency encoders of the main experiment $(p>.10)$. An examination of mean performance on the relative frequency judgment task indicated that this group of subjects was comparable to those serving in the main experiment (mean $=31.6, \mathrm{SD}=2.7)$. Thus, a significant correlation was obtained between frequency judgment ability and the strategy identification task when a group of subjects was instructed to employ inferred frequency encoding activities on the latter task. This result lends support to our notion that subjects reporting such activities in the main experiment were in fact utilizing frequency discrimination processes in performing the strategy identification task as well as the verbal discrimination learning task.

\section{GENERAL DISCUSSION}

Frequency theory (Ekstrand et al., 1966) states that performance in the verbal discrimination task is based on subjects' discrimination of subjective frequency differentials within pairs. We have tentatively concluded from Experiment 2 that this formulation does not apply across the board with adults. While the correlational analysis indicated that many of our adult subjects utilized frequency cues in the verbal discrimination task, the analysis also indicated that others spontaneously adopted alternative strategies (e.g., imaginal or verbal strategies) which led them to utilize attributes other than frequency. It should be noted, however, that only a relatively small number of subjects reported using such strategies.

In this regard, it is important to note that frequency theory does not assert that frequency is the only attribute that can be used in verbal discrimination learning, but rather that it is the predominant attribute under "normal" circumstances (i.e., under conditions which do not bias subjects toward some other attribute). This latter notion was supported in our experiment with adults, where a majority of subjects were inferred to have utilized frequency. And it was certainly supported in the case of fifth- and six th-grade children, where we found no evidence that other attributes were utilized in the control version of the verbal discrimination learning task.

Finally, it should be mentioned that the usefulness of our combined experimental-correlational paradigm in resolving theoretical issues in discrimination learning has been demonstrated in this and in previous research. It seems reasonable to expect that variations of the paradigm could be used to uncover performance attributes in other learning and memory tasks as well. In this regard, a number of extensions of the paradigm to recognition situations involving semantically integrated materials appear imminent (see also Ghatala \& Levin, 1976).

\section{REFERENCES}

Ekstrand. B. R., Wallace, W. P., \& Underwood, B. J. A frequency theory of verbal-discrimination learning. Psychological Review, 1966, 73, 566-578.

Flexser, A. J., \& Bower, G. H. Further evidence regarding instructional effects on frequency judgments. Bulletin of the Psychonomic Society, 1975, 6. 321-324.

Ghatala. E. S., \& Levin, J. R. Children's recognition memory processes. In J. R. Levin \& V. L. Allen (Eds.), Cognitive learning in children: Theories and strategies. New York: Academic Press, 1976.

Ghatala, E. S., Levin, J. R., \& Subroviak, M. J. Rehearsal strategy effects in children's discrimination learning: Confronting the crucible. Joumal of Verbal Learning and Verbal Behavior, 1975, 14, 398-407.

Levin, J. R., \& GhatalA, E. S. More about rehearsal strategy effects in children's discrimination learning. Journal of Experimental Psychology: Human Learning and Memory. 1976, 2, 381-390.

Levin, J. R., Ghatala, E. S., DeRose, T. M., Wilder, L., \& Norton, R. W. A further comparison of imagery and vocalization strategies in children's discrimination learning. Journal of Educational Psychology, 1975, 67, 141-145.

Levin, J. R.. Ghatala, E. S., Wilder, L., \& Inzer, E. Imagery and vocalization strategies in children's verbal discrimination learning. Journal of Educational Psychology, 1973, 64, 360-365.

Rose, R. J., \& Rowe, E. J. Effects of orienting task and spacing of repetitions on frequency judgments. Journal of Experimental Psychology: Human Learning and Memory. 1976, 2, 142-152.

Rowe, E. J.. \& CAKE, L. J. Imagery and sentence mediators in verbal discrimination learning. Memory \& Cognition. 1974, 2, 169-175.

Rowe, E. J., \& PaIvio, A. Imagery and repetition instructions in verbal discrimination and incidental paired-associate learning. Journal of Verbal Leaming and Verbal Behavior, 1971, 10, 668-672.

Underwood, B. J., \& Freund, J. S. Two tests of a theory of verbal discrimination learning. Canadian Journal of Psychology, 1968, 22, 96-104.

UNDERWOOD, B. J. Individual differences as a crucible in theory construction. American Psychologist, 1975, 30. 128-134.

WILDER, L. Spoken rehearsal and verbal discrimination learning. Speech Monographs, 1971, 38, 113-120.

(Received for publication July 16, 1976; revision accepted September 28,1976 .) 